\title{
A stable realization of apodization filtering applied to noise SAR and SAR range sidelobe suppression
}

\author{
Xin $\mathrm{Wu}^{1,2^{*}}$, Yanfei Wang ${ }^{1}$, Shu Li $i^{1,2}$ and Chang Liu ${ }^{1}$
}

\begin{abstract}
Pulse response of radar system always suffers from high sidelobe level resulting in resolution degradation. Investigated here is a sidelobe suppression method based on apodization filtering technique for range responses of synthetic aperture radar (SAR) and noise SAR systems. The core of apodization filtering is finding an appropriate filtering vector in time domain. Compared with original apodization filtering, the proposed method could be realized stably because it could get correct filtering vector efficiently. This method contains three important steps: constructing coefficient matrix and desired response vector; performing ill-posed analysis; and solving equation to find filtering vector. In these steps, convolution kernel method is adopted to construct coefficient matrix; spectral condition is introduced as an indicating function for ill-posed analysis; and total variation method is used to resolve ill-posed equation for getting filtering vector. Elaborate theoretical derivation is presented to demonstrate the feasibility of this method. In order to test its effect, simulation experiments are implemented. Simulation results show that there is a great suppression of range sidelobes after processed by this method. With increasing filter length, the performance of filtered output is improved but time cost is increasing correspondingly. Furthermore, the proposed method is also effective with noise disturbance.
\end{abstract}

Keywords: apodization filtering, sidelobe suppression, stability, noise synthetic aperture radar

\section{Introduction}

Synthetic aperture radar (SAR) is a technique in which backscattered microwave pulses are collected at different positions to synthesize a long antenna. It is able to get radar images with high resolution [1]. To obtain radar images of the detected regions, SAR first transmits linear frequency modulation signals, and then performs pulse compression along range and azimuth directions for echo signals. The pulse responses of SAR along range and azimuth are both sinc functions with high levels of sidelobes [2], thus, it is important to reduce sidelobes for high-resolution SAR images.

Noise SAR system combines random noise radar with SAR technique holding both advantages of the two individuals. Random noise radar refers to radar whose transmitted signal is a microwave noise or is modulated by noise source. It has excellent electronic counter

\footnotetext{
* Correspondence: abcd1002@tom.com

'Institute of Electronics, Chinese Academy of Sciences, Beijing 100190, China Full list of author information is available at the end of the article
}

countermeasure capability, very low probability of intercept, high electro-magnetic compatibility, good counter electronic support measure capability, and ideal 'thumbtack' ambiguity function [3]. In [4-7], basic principles and typical noise radar systems are presented with further analysis. Noise radar was originally developed with homodyne receivers for short-range applications [5]. In current, it has been used in diverse areas, such as SAR and inverse synthetic aperture radar [8-10], collision warning, altimetry, ground penetration detection of buried objects, and Doppler estimation [11-13]. Recently, depending on random noise radar system, through-wall detection technique is developing fast for anti-terrorism and earthquake rescue [14,15].

Noise SAR makes random noise radar acquire the capability to imaging, meanwhile it improves the performance of anti-jamming for SAR system due to its truly random transmission signal [16]. It has become a useful remote sensor for military and civil missions, such as high-resolution land mapping, wave height monitoring, 
forest inventory, etc. Noise SAR imaging has been studied a lot in recent years. Experimental researches focusing on noise SAR imaging algorithms have been implemented in practice $[8,9,17]$. In noise SAR imaging, the system also suffers from high sidelobes which leads to resolution degradation for images. Target detection is severely deteriorated by high sidelobe level, especially in subsurface profiling application. Therefore, sidelobe reduction is meaningful to obtain correct output responses and high-quality noise SAR images.

Amplitude weighting is usually applied to depress sidelobes by multiplying a window function to the response in frequency domain [18]. However, this method always results in mainlobe broadening. Some image domain deconvolution methods such as CLEAN technique are investigated to suppress sidelobes [19]. These methods require some special conditions, for instance, the brightest spot in image must be a true scatterer, so that their applications are limited. Spatially variant apodization (SVA) is another method for sidelobe suppression. It is able to control sidelobes of SAR images effectively by applying sequential nonlinear operations to complex-valued SAR imagery [20]. This method is originally proposed with requirement that the data should be sampled at an integer multiple of Nyquist frequency, but now it can be used to suppress sidelobes in any case of Nyquist sampling rates [21].

Signal processing of noise SAR is different from general SAR because random transmitted signal has no specific analytic expression. As an alternative to matched filtering, correlation operation is implemented directly in time domain to perform pulse compression along range direction for noise SAR imaging. The range profiles are formed depending upon delay line which provides changed time delays to correlate with echoed signals by directly sampling in time domain. Amplitude weighting cannot be applied to noise SAR imaging because it should be operated in frequency domain. Image domain deconvolution methods such as CLEAN technique are not good choices either because of the required conditions. Using a set of different short filters optimized for every pixel, SVA is indeed an effective method. Applying SVA to noise radar could be an interesting research, nevertheless, this article studies about another method. To control the output sidelobes of random noise radar, apodization filtering technique is proposed as a special algorithm [22]. Its effect has been proved by the application to ground penetration of random noise radar. As a new technique, some problems still to be resolved. For instance, realization process is not stable enough, mathematic model is not accurate adequately, applications are not extensive, restrictive conditions are excessive, etc. Therefore, further investigations are required to perfect it.
In this article, a stable realization of apodization filtering (SRAF) is proposed, which is a modified method to original realization. It is able to depress sidelobes significantly and improve the stability of apodization filtering. SRAF is not only carried out on range sidelobe control of correlation output for random noise SAR, but also implemented in matched filtering output for general SAR. This article is organized as follows: the basic principle of apodization filtering technique is first reviewed in Section 2. With elaborate theoretical derivation and specific mathematical representations, three important steps of SRAF are presented in Section 3. Applications of SRAF in range sidelobe suppression for SAR and noise SAR are demonstrated in Section 4. In this section, simulation results and some important data are further analyzed. And finally a summary and some conclusions are provided in Section 5.

\section{Principle of apodization filtering}

Apodization filtering technique is originally proposed to control sidelobes for random noise radar. Its basic principle is simply introduced in this section. After getting a filtering function in time domain, output response should be filtered by this function to achieve sidelobe suppression.

In conventional Fourier analysis, frequency domain data are multiplied by window functions. After inverse Fourier transforming the multiplication data, the sidelobes of output are effectively suppressed. According to convolution theorem, a multiplication in frequency domain is equivalent to a convolution in time domain, so that a corresponding filter in time domain can be obtained. The convolution operation is expressed as

$$
G(\tau)=A(\tau) \otimes F(\tau)
$$

Range resolution mainly depends on the point spread function (PSF) of radar system. $A(\tau)$ is the PSF with high sidelobe level, and it is called original output response. $G(\tau)$ is prospective output response determined based on ideal case. $F(\tau)$ is goal filtering function in time domain, and $\otimes$ denotes convolution operation. In general, the digitized of Equation (1) is adopted, and it is written in matrix form as

$$
K F=G
$$

where $\boldsymbol{K}$ is coefficient matrix, $\boldsymbol{F}$ and $\boldsymbol{G}$ are filtering vector and desired response vector which are digitized forms of $F(\tau)$ and $G(\tau)$, respectively. The ultimate objective is getting an appropriate filtering vector $\boldsymbol{F}$ that makes the sidelobes of output response be effectively controlled and the energy of mainlobe be preserved well. In realization of this method, filter length, which is the dimension of goal filtering vector $\boldsymbol{F}$, should be 
determined in first, and then coefficient matrix $\boldsymbol{K}$ and desired response vector $\boldsymbol{G}$ can be constructed. Finally, a practicable method should be applied to solving Equation (2) for getting vector $\boldsymbol{F}$ based on $\boldsymbol{K}$ and $\boldsymbol{G}$. The general steps of apodization filtering are shown in Figure 1.

\section{Sidelobe suppression algorithm based on SRAF}

From the previous section, finding filtering vector $\boldsymbol{F}$ is the key of apodization filtering. As can be seen from Equations (1) and (2), getting $\boldsymbol{F}$ is essentially to solve a first kind integral equation which generally represents ill-posed system, so that the stability of adopted method is as important as its efficiency. Original realization of apodization filtering (ORAF) is introduced in [22]. It has successfully been implemented for random noise radar in order to suppress the range sidelobes by applying projection method to resolve the ill-posed problem. However, its stability is not enough because inappropriate solution may be obtained. Improper filtering can lead to obvious distortion of output response. Therefore, a modified method SRAF is proposed in this article to acquire correct filtering vector with following processing details.

\subsection{Coefficient matrix and desired response vector}

As shown in Figure 1, coefficient matrix $K$ and desired response vector $\boldsymbol{G}$ should be determined before resolving Equation (2). Approaches to formations of $\boldsymbol{K}$ and $\boldsymbol{G}$ are presented in this section.

Comparing Equations (1) and (2), product of matrix $\boldsymbol{K}$ and vector $\boldsymbol{F}$ is equivalent to convolution operation $A(\tau)$ $\otimes F(\tau)$. This means that the convolution should be expressed as the product. Therefore, $\boldsymbol{K}$ is a convolution

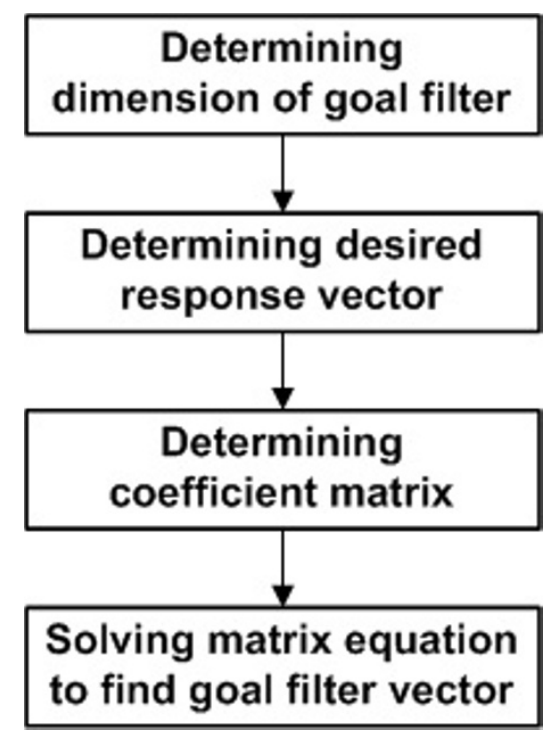

Figure 1 General steps of apodization filtering. matrix, whose rows are reversed, conjugated, and timeshifted versions of $A(\tau)$ 's sampling. Therefore, convolution kernel approach can be introduced to get $\boldsymbol{K}$. The sampled version of $A(\tau)$ is expressed as

$$
A=[a_{1} a_{2} \ldots \underbrace{a_{m_{1}} a_{m_{2}} \cdots a_{m_{k}}}_{\text {mainlobe }} \cdots a_{m-1} a_{m}]_{m \times 1}^{\mathrm{T}}
$$

where $m$ is the dimension of $A$, and $a_{m_{i}}(i=1,2, \ldots, k)$ are the elements corresponding to mainlobe. Filtering function $\boldsymbol{F}$ can be denoted as

$$
\boldsymbol{F}=\left[f_{1} f_{2} \ldots f_{n}\right]_{n \times 1}^{T}
$$

where $n$ is filter length that should be determined in advance. Based on convolution kernel approach, matrix $\boldsymbol{K}$ can be constructed by Equation (5).

$$
\boldsymbol{K}=\left[\begin{array}{ccccc}
a_{1} & & & \\
a_{2} & a_{1} & & \\
\vdots & a_{2} & \ddots & \\
a_{m} & \vdots & & a_{1} \\
& a_{m} & a_{2} \\
& & \ddots & \vdots \\
& & & a_{m}
\end{array}\right]_{(m+n-1) \times n}
$$

Elements not represented in Equation (5) are zero. As shown in Equation (5), matrix $\boldsymbol{K}$ is determined by original response and filter length together. Formulated by Equation (5), coefficient matrix $\boldsymbol{K}$ is guaranteed to accomplish the convolution operation without approximation. Compared with ORAF, it has adequate accuracy of matrix model with no restriction on filter length.

In an ideal output response, sidelobes are absolutely suppressed. Thus, desired response $\boldsymbol{G}$ is selected as follows: set the amplitude of all sidelobe points to be zero, and set the amplitude of mainlobe points to be the same as original response $\boldsymbol{A}$. According to this selection principle, vector $\boldsymbol{G}$ is of the form

$$
G=[0 \ldots 0 \underbrace{a_{m_{1}} a_{m_{2}} \ldots a_{m_{k}}}_{\text {mainlobe }} 0 \ldots 0]_{(m+n-1) \times 1}^{T}
$$

where $a_{m_{i}}(i=1,2, \ldots, k)$ should be located at the center of $\boldsymbol{G}$.

\subsection{III-posed analysis}

After matrix $\boldsymbol{K}$ and vector $\boldsymbol{G}$ have been determined, a direct solution for Equation (2) could be achieved by least squares method. However, if Equation (2) represents an ill-posed system, direct solution is practically impossible. Therefore, ill-posed analysis is necessary to decide which method is feasible to obtain correct 
solution. Whether an equation is ill posed or not depends on the coefficient matrix, so that matrix $K$ is further analyzed starting with singular value decomposition (SVD) involved.

With implementation of SVD, matrix $\boldsymbol{K}$ is expressed as

$$
\boldsymbol{K}=\boldsymbol{U} \boldsymbol{\Sigma}_{m+n-1, n} \boldsymbol{V}^{T}
$$

where $\boldsymbol{U}$ and $\boldsymbol{V}$ are both unit orthogonal matrices with sizes of $(m+n-1) \times(m+n-1)$ and $n \times n$, respectively; and $\Sigma_{m+n-1, n}$ is a matrix of $(m+n-1) \times n$, whose representation is shown as Equation (8)

$$
\boldsymbol{\Sigma}_{m+n-1, n}=\left[\begin{array}{cccc}
\sigma_{1} & & & \\
& \sigma_{2} & & \\
& & \ddots & \\
& & & \sigma_{n} \\
& & 0 & \\
& & \vdots
\end{array}\right]_{(m+n-1) \times n}
$$

where $\sigma_{i}(i=1,2, \ldots, n)$ denote the non-zero singular values of matrix $K$ with degressive order from $\sigma_{1}$ to $\sigma_{n}$.

The least squares solution (LSS) of Equation (2) is in the form of

$$
F_{\text {LSS }}=\left(K^{T} K\right)^{-1} K^{T} G
$$

After inserting Equations (7) and (8) into Equation (9), $\boldsymbol{F}_{\mathrm{LSS}}$ is rewritten as

$$
\boldsymbol{F}_{\mathrm{LSS}}=V\left[\begin{array}{ccccc}
\sigma_{1}^{-1} & & & & \\
& \sigma_{2}^{-1} & & & \\
& & \ddots & 0 & \cdots \\
& & & \sigma_{n}^{-1}
\end{array}\right]_{n \times(m+n-1)} \quad \boldsymbol{U}^{T} \boldsymbol{G}=\sum_{i=1}^{n} \frac{\boldsymbol{u}_{i}^{T} \boldsymbol{G}}{\sigma_{i}} \boldsymbol{v}_{i}
$$

where $\boldsymbol{u}_{i}$ and $\boldsymbol{v}_{i}$ denote the column vectors of matrices $U$ and $V$, respectively.

In the denominator of Equation (10), singular values of matrix $K$ highly influence the stability of solution $\boldsymbol{F}_{\mathrm{LSS}}$. If matrix $\boldsymbol{K}$ has very small singular values, a little variance from matrix $G$ will cause serious fluctuation of solution $\boldsymbol{F}_{\mathrm{LSs}}$. That is to say, small singular value of $\boldsymbol{K}$ is the reason behind the ill-posed state of Equation (2). Therefore, a function which depends on singular values of $\boldsymbol{K}$ could be selected to evaluate whether Equation (2) represents an ill-posed system. This function is defined as Equation (11)

$$
\kappa(K)=\frac{\sigma_{\max }}{\sigma_{\min }}=\frac{\sigma_{l}}{\sigma_{n}}
$$

Determined by the quotient of the maximum singular value to the minimum one, $\kappa(\boldsymbol{K})$ is called spectral condition of matrix $\boldsymbol{K}$. If the value of $\kappa(\boldsymbol{K})$ is very large with the form of $10^{n}(n>6)$, Equation (2) is confirmed to be ill posed.

\subsection{Approach to getting filtering vector}

Based on ill-posed analysis result, a suitable method for getting filtering vector should be adopted. In essence, this problem is to solve a first kind integral equation which generally represents ill-posed system. In this section, total variation (TV) method is introduced to resolve the problem.

Depending on the peculiarity of equation, ill-posed problem is generally resolved via constructing an additional regularizing function to resume stability $[23,24]$. In this article, a solution vector with finite length is utilized to approach the true solution which makes plentiful non-zero elements of original response become zero, so that the solution is likely to have several discontinuous points. TV is a specific regularization method with advantage of non-restricting solution to be smooth [25]. It is capable of resolving the ill-posed problem in this article because it is able to retain the discontinuous boundary of solution.

TV method focuses on transforming Equation (2) to an alternative function $\boldsymbol{J}^{\alpha, \beta}(\boldsymbol{F})$ with well stability [26], whose representation is Equation (12).

$$
J^{\alpha, \beta}(F)=\frac{1}{2}\|\mathbf{K F}-G\|^{2}+\alpha J_{\beta}(F)
$$

where $\|\cdot\|$ represents $\ell_{2}$ norm; $\boldsymbol{J}_{\beta}(\boldsymbol{F})$ is regularizing function to resume the stability of Equation (2), and it is expressed as

$$
J_{\beta}(F)=\int_{\mathrm{U}} \sqrt{|\nabla F(\tau)|^{2}+\beta} \mathrm{d} \tau
$$

where $\mathbf{U}$ is the support domain of $F(\tau) . \alpha$ and $\beta$ are the regularization and regulated parameters with positive values. The two parameters have influences on the stability of solving ill-posed equation.

As a function relating to $\boldsymbol{F}, \boldsymbol{J}^{\alpha, \beta}(\boldsymbol{F})$ should be minimized to search an adequate filtering vector with variable $\boldsymbol{F}$. Expected filter is the vector $\boldsymbol{F}$ that makes Equation (12) to achieve minimum value. For obtaining this filter, fixed-point iteration algorithm is implemented to accomplish the minimizing processing. With initial guess solution $\boldsymbol{F}^{0}=[0 \ldots 0]_{n \times 1}^{T}$, equations as Equation (14) should be iteratively resolved.

$$
\left[\boldsymbol{K}^{*} \boldsymbol{K}+\alpha L_{0}\left(\boldsymbol{F}^{m}\right)\right] \boldsymbol{F}^{m+1}=\boldsymbol{K}^{*} \boldsymbol{G}
$$

where $\boldsymbol{K}^{*}$ is the adjoint matrix of $\boldsymbol{K}$, the superscript of $\boldsymbol{F}$ denotes iterative steps, and $L_{0}\left(F^{m}\right)$ represents ellipse differential coefficient operator. If operator $L\left(F^{m}\right)$ is operated at vector $\boldsymbol{F}$, corresponding representation is 
defined as Equation (15).

$$
\mathcal{L}\left(F^{m}\right) F=-\nabla \cdot\left(\frac{1}{\sqrt{\left|\nabla F^{m}\right|^{2}+\beta^{2}}} \nabla F\right)
$$

where $\nabla \cdot(f)$ is equal to $\operatorname{div}(f)$ denoting the divergence of vector $\boldsymbol{f}$, and $\nabla \boldsymbol{f}$ means the gradient of $\boldsymbol{f}$. The digitized version of Equation (15) should be adopted, which is approximately expressed as

$$
\left\llcorner\left(F^{m}\right) \boldsymbol{F}=\tilde{\boldsymbol{D}}^{T} \boldsymbol{Q}_{m}^{-1} \tilde{\boldsymbol{D}} \boldsymbol{F}\right.
$$

where $\boldsymbol{Q}_{m}$ is a diagonal matrix corresponding to the digitized version of $\sqrt{\left|\nabla F^{m}\right|^{2}+\beta^{2}}$; and subscript $m$ represents iterative steps. Matrices $\boldsymbol{Q}_{m}$ and $\tilde{D}$ can be constructed by Equations (17) and (18), respectively.

$$
\begin{aligned}
& Q_{m}(k, k)=\sqrt{\left[F^{m}(k)-F^{m}(k-1)\right]^{2}+\beta^{2}} \\
& Q_{m}(1,1)=\sqrt{\left[F^{m}(1)\right]^{2}+\beta^{2}} \\
& \tilde{D}=\left[\begin{array}{ccccc}
-a & & & & \\
b & -a & & & \\
& b & \ddots & & \\
& & \ddots & \ddots & \\
& & & b & -a
\end{array}\right]_{n \times n}
\end{aligned}
$$

In Equation (17), $k=2,3, \ldots, n$ represents the $k$ th element of $\boldsymbol{F}^{m}$. Parameters $a$ and $b$ in Equation (18) are
0.665 and 0.1755 , which are determined by minimizing function $f(a, b)=64\left(a^{2}+2 b^{2}-0.5\right)^{2}+224(0.125-a b)^{2}+98 b^{4}$ $[26,27]$. Then, Equation (14) can be digitized as Equation (19)

$$
\left[\boldsymbol{K}^{T} \boldsymbol{K}+\alpha \tilde{\boldsymbol{D}}^{T} \boldsymbol{Q}_{m}^{-1} \tilde{\boldsymbol{D}}\right] \boldsymbol{F}^{m+1}=\boldsymbol{K}^{T} \boldsymbol{G}
$$

Equation (19) is easy to solve by traditional conjugate gradient method.

We can successively obtain $\boldsymbol{F}^{1}, \boldsymbol{F}^{2}, \boldsymbol{F}^{3}, \ldots$ until $\boldsymbol{F}^{n}$ converging to threshold through iterative resolving Equation (19). In theory, vector $\boldsymbol{F}^{n}$ is able to satisfy Equation (20) as follows.

$$
\lim _{n \rightarrow \infty}\left\|F^{n}-F^{n-1}\right\|=0
$$

In order to decide whether current solution is converged, the norm of difference between the solution vectors of current and former iterations is an obvious indicator. Threshold for the norm must be small enough to ensure that the iterative algorithm converging to a desired solution. In general, threshold is selected less than $10^{-2}$. Let $\boldsymbol{F}=\boldsymbol{F}^{n}$ as filtering vector, the result of multiplying coefficient matrix $\boldsymbol{K}$ with filtering vector $\boldsymbol{F}$ is filtered output with sidelobes suppression. The process of solving ill-posed equation for filtering vector is shown as Figure 2.

There is an important issue that should be concerned with filtered output after filtering by vector $\boldsymbol{F}$. Since $m$ $+n$ - 1 dimension is much longer than $m$, the length of filtered output must be revised to be consistent with

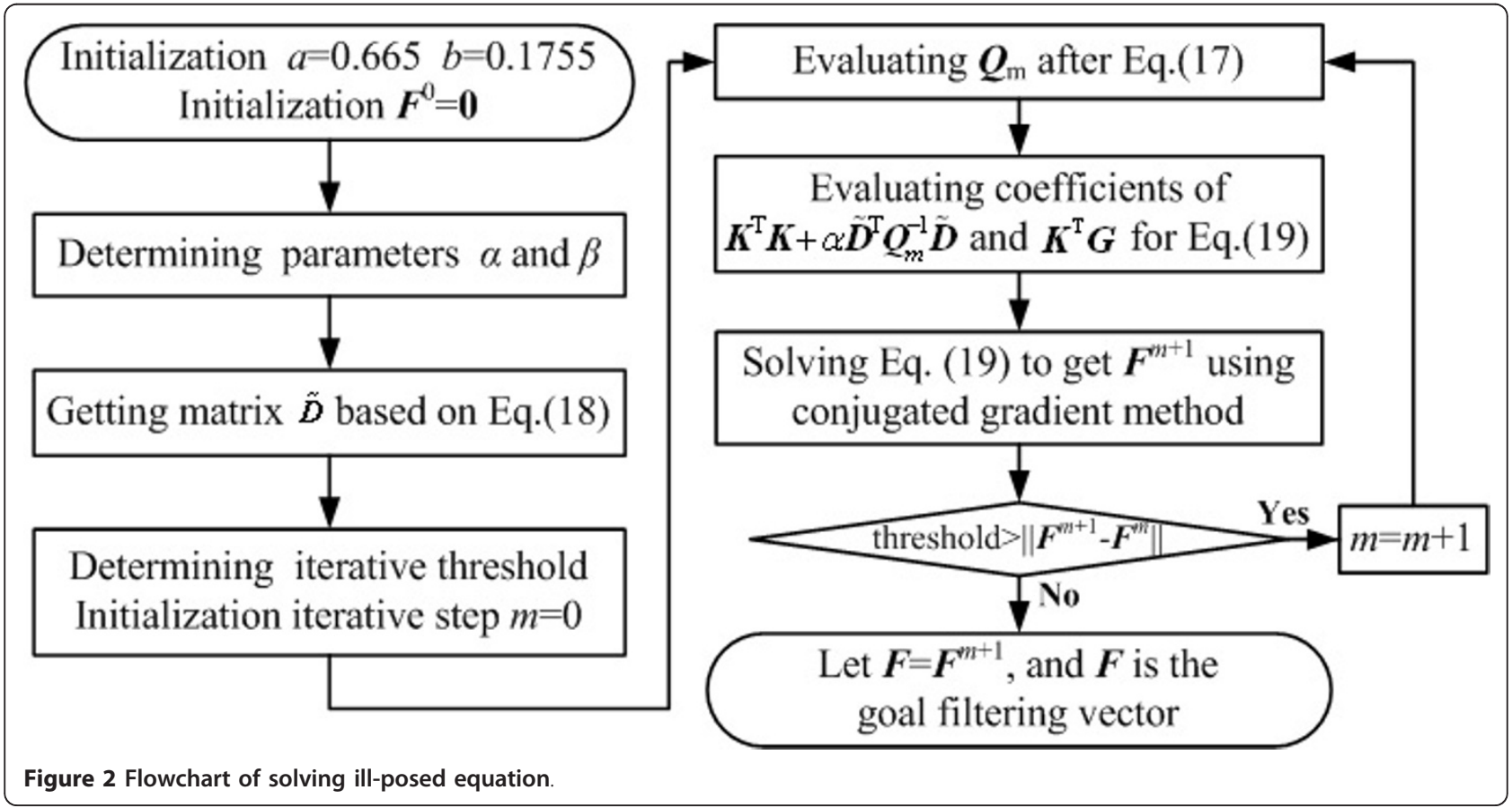


original response $A$. For filtered output, the points at two sides can be abandoned because their values are so little that hardly include useful information after sidelobe suppression. Therefore, revision for length could be performed as follow: with number of $m$, the points in the middle of filtered output are saved as final output; each $(n-1) / 2$ points in the two sides are deleted. After length revision, final filtered output is obtained, and the filtering process of SRAF is accomplished with flowchart shown in Figure 3.

Here, it is necessary to present how to determine parameters $\alpha$ and $\beta$ that influence the stability of Equation (12). For optimal value of $\alpha$, function $\phi(\lambda)$ is formed as Equation (21).

$$
\varphi(\lambda)=\frac{1}{m} \sum_{i=1}^{m} \frac{|G(i)|}{\lambda|A(i)|^{2}}-\|\varepsilon\|^{2}
$$

In Equation (21), $\boldsymbol{G}(i)$ is composed of the $m$ middlemost elements after Fourier transforming to desired response $\boldsymbol{G}$, and $\boldsymbol{A}(i)$ is the Fourier transform of original response $A$. Through one dimension searching, it is easy to find an optimal $\lambda$ that makes $\phi(\lambda)$ be zero because $\phi(\lambda)$ is a single descending function. Then, the optimal $\alpha$ can be estimated by $\alpha=q / \lambda$, where $q$ is the mean of $Q_{m}$. In $[26,28]$, the methods about selecting regularization parameter are provided. With small and positive value, parameter $\beta$ is regulated to ensure that regularizing function $\boldsymbol{J}_{\beta}(\boldsymbol{F})$ is differentiable at $\mathbf{F}=\mathbf{0}$. In practice, it is estimated by experience and iterative tests.

\section{Experimental results and performance analysis}

In order to test the effect of SRAF, simulations are performed and the results are provided in this section. SRAF is not only applied to range sidelobes suppression of correlation output for noise SAR, moreover, it is implemented to control the sidelobes of matched filtering output for general SAR.

\subsection{Implementation of sidelobe suppression based on SRAF}

In this section, simulation results are provided to illustrate the effect of SRAF applied to range sidelobe suppression. System parameters selected for simulations are shown in Table 1. Two situations $(\mathrm{SNR}=\infty$ and SNR $=$ $20 \mathrm{~dB}$ ) are considered in all simulations. In following figures, black solid line denotes the results without noise disturbance while color dash line represents the consequences when SNR is $20 \mathrm{~dB}$.

Figure 4 shows the results corresponding with correlation output of noise SAR. Figure $4 \mathrm{~A}$ is the original

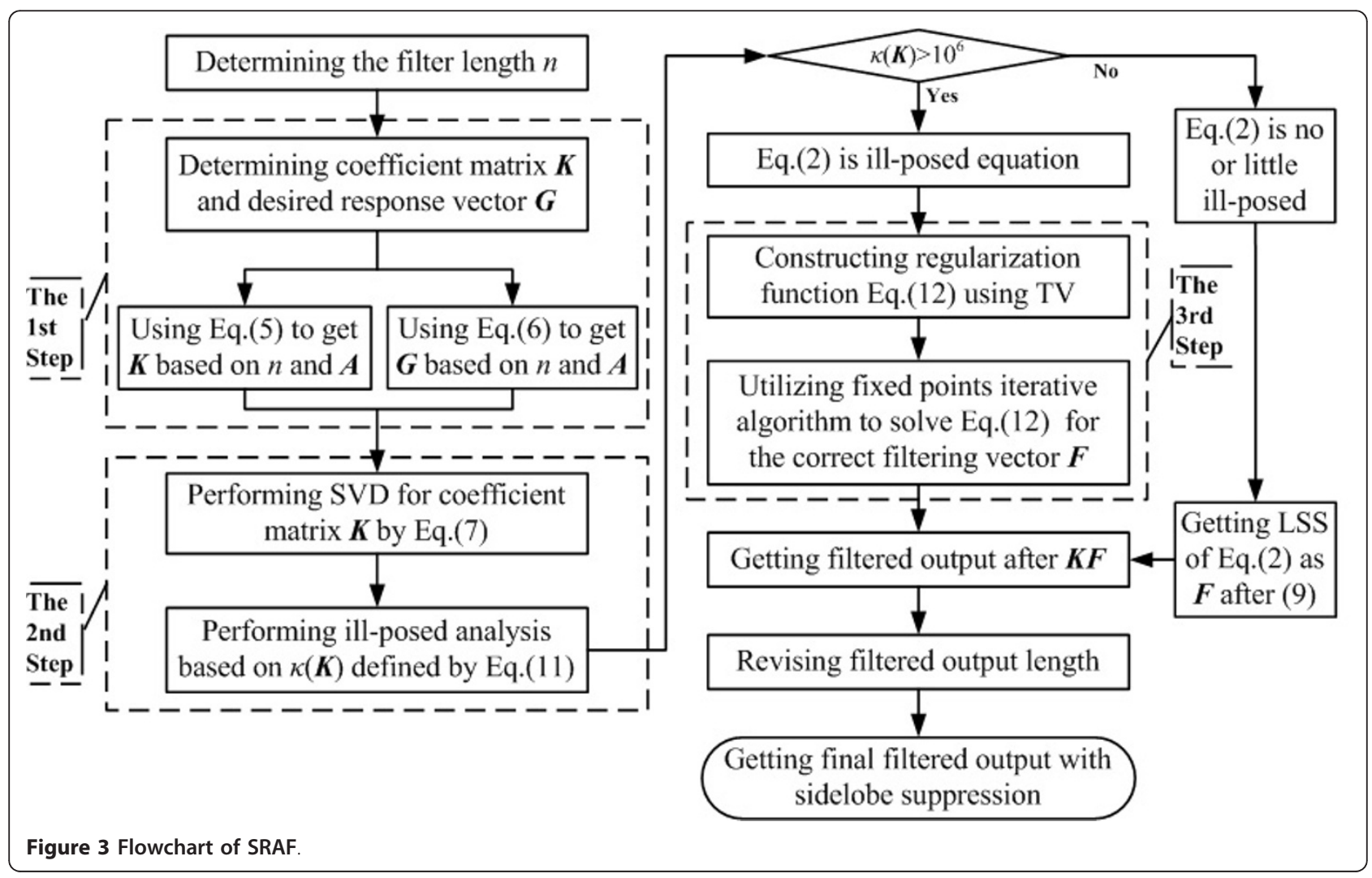


Table 1 System parameters

\begin{tabular}{lll}
\hline Parameter & Value & \\
\cline { 2 - 3 } & Range correlation (noise SAR) & Matched filtering (general SAR) \\
\hline Carrier frequency & $1 \mathrm{GHz}$ & $1 \mathrm{GHz}$ \\
transmitted signal bandwidth & $51.2 \mathrm{MHz}$ & $15 \mathrm{MHz}$ \\
Pulse duration & $10 \mu \mathrm{s}$ & $2 \mu \mathrm{s}$ \\
Sampling time interval & $19.5 \mathrm{~ns}$ & $6.67 \mathrm{~ns}$ \\
\hline
\end{tabular}

output without sidelobe control. Figure 4B-D is the outputs filtered by SRAF with filter length 900, 1500, and 2700 , respectively. The peak sidelobe level ratio (PSLR) and integrated sidelobe level ratio (ISLR) of original and filtered outputs are provided in the figures. As can be seen from Figure 4, greater sidelobe reduction is achieved with increasing filter length.

Under realistic conditions, the power of noise disturbance should be increased. Figure 5 shows the results after SRAF filtering with filter length 2700 in low SNR.
Figure 5A, B corresponds with conditions of SNR = $-0.5 \mathrm{~dB}$ and $\mathrm{SNR}=-10 \mathrm{~dB}$, respectively. As shown in Figure 5, although the performance of filtered output is not as good as that of Figure 4, sidelobes are also suppressed effectively with faithful preservation of mainlobe power.

In the second simulation, SRAF is used in matched filtering output of general SAR to control range sidelobes. Figure 6 demonstrates the simulation results. Figure 6A is the original output without sidelobe suppression.
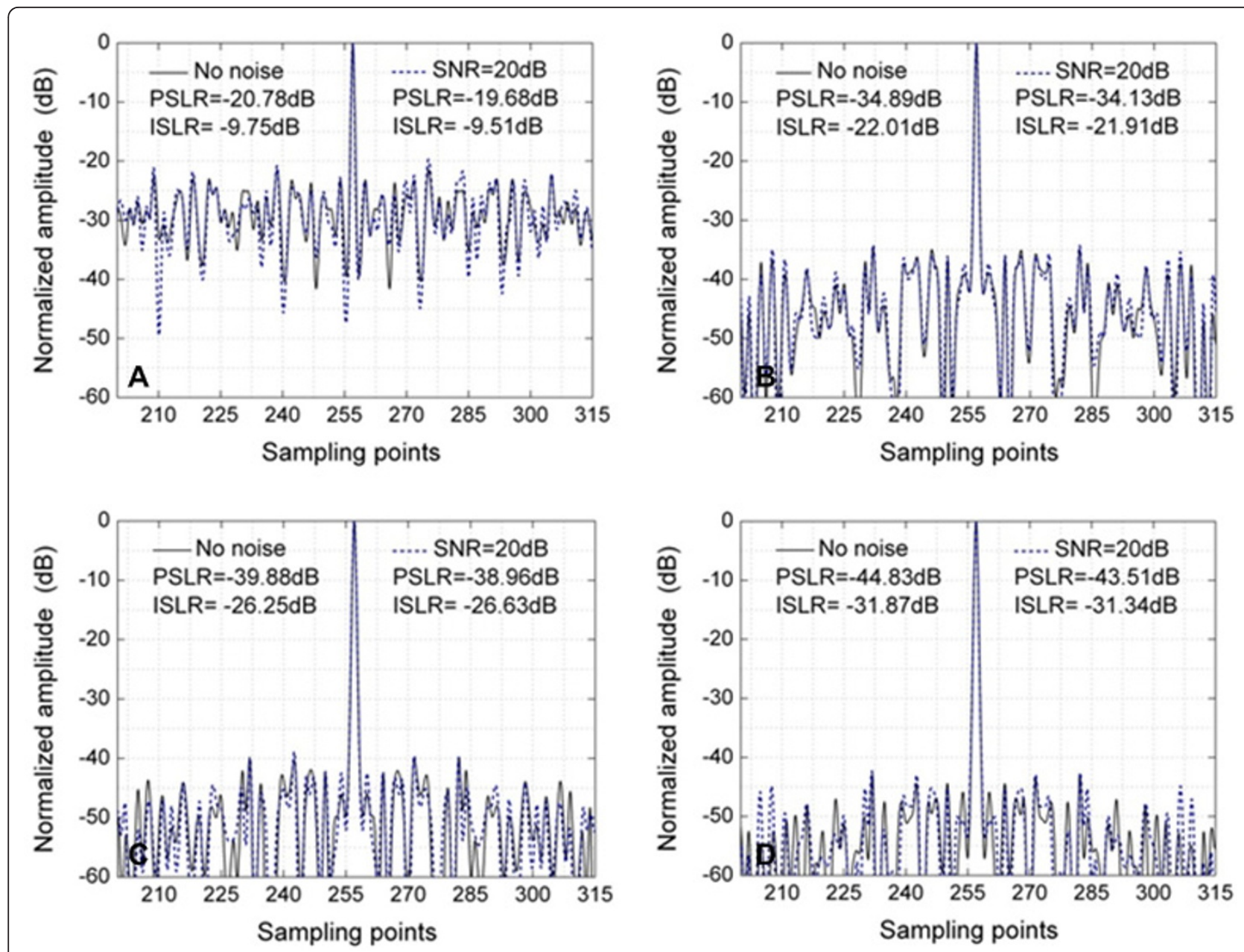

Figure 4 Correlation outputs before and after sidelobe suppression. The outputs relating to SNR $=\infty$ are denoted by black solid line; and the outputs relating to $S N R=20 \mathrm{~dB}$ are denoted by color dash line. (A) Original outputs without sidelobe suppression. (B) Outputs filtered by SRAF with filter length 900. (C) Outputs filtered by SRAF with filter length 1500. (D) Outputs filtered by SRAF with filter length 2700. 

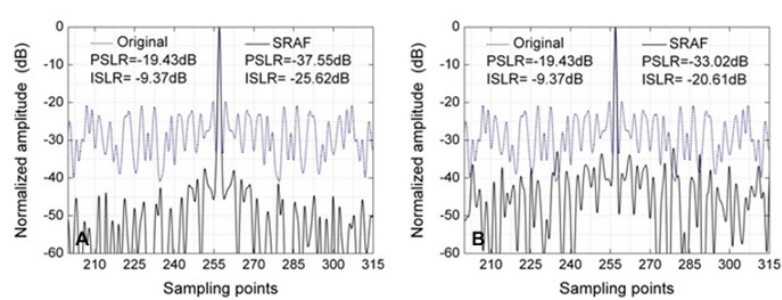

Figure 5 Outputs of SRAF in noise disturbance with filter length $\mathbf{2 7 0 0}$. The original outputs are described by black solid line; and the sidelobe suppressed outputs are described by color short dot line. (A) $S N R=-0.5 d B$. (B) $S N R=-10 d B$.

Figure $6 \mathrm{~B}, \mathrm{C}$ is the outputs after SRAF filtering with filter lengths 500 and 800 , respectively. To compare with SRAF, traditional amplitude weighting is also adopted to reduce sidelobes. Figure 6D, E presents the results weighted by Hamming and Chebyshev windows. In the case of ideal SNR, results of these algorithms are showed in Figure 6F with relevant PSLR, ISLR, and IRW. As Figure 6 revealed, SRAF is capable of suppressing sidelobes with preservation of mainlobe energy; whereas Hamming and Chebyshev windows can control sidelobes at the cost of mainlobe broadening. After SRAF filtering, the performance of outputs has no obvious fluctuation while SNR is depressed from infinite to $20 \mathrm{~dB}$. In contrast, the outputs weighted by Hamming and Chebyshev windows are degraded seriously when SNR is $20 \mathrm{~dB}$.

Figure 7 is an example of matched filtering for three targets that are close to each other in range. Two of these targets have equal radar cross section, whereas the other is a weak target with much lower radar cross section. Figure 7A is the original output after matched filtering. The weak target is obscured by sidelobes of strong targets. Figure 7B, C shows the results weighted by Hamming and Chebyshev windows. Though sidelobes are both suppressed, but the three targets are not separated thoroughly because of mainlobe broadening. In Figure 7B, the weak target could be detected ambiguously, however, it is difficult to separate the two strong targets which are close each other. In Figure 7C, three targets are combined together without any discrimination. Figure 7D shows the result filtered by SRAF with filter length 1000 . In contrast to Figure $7 \mathrm{~B}, \mathrm{C}$, all targets are detected due to sidelobe reduction and mainlobe energy preservation. Furthermore, Figure 7 also shows that influence of noise disturbance to SRAF is much lower than that to amplitude weighting. Serious performance degradations are observed in Figure 7B, C.

\subsection{Analysis of experimental data}

To evaluate the performance and efficiency of proposed method, some important simulation data are presented for further analysis. All data in this section are relevant to the simulations in Section $4.1(\mathrm{SNR}=\infty)$. In the implementation of SRAF, threshold of iterative convergence is $10^{-5}$; parameters $\alpha$ and $\beta$ are $2 \times 10^{-6}$ and 0.01 .

With filter length increasing, the extremal singular values and spectrum conditions of matrix $K$ are shown in Table 2 to analyze if the corresponding equations represent ill-posed system. As Table 2 shown, $\kappa(\boldsymbol{K})$ fluctuates about $10^{10}$ which is large enough to stand for illposed system. When filter length is increasing, ill-posed degree for the equation becomes more serious, so that least square method is infeasible to get solution. The iterative steps and running times about solving equations are presented in Table 3 to evaluate computational efficiency. Table 3 reveals that filtering vector can be obtained within several iterative steps, thus the process of SRAF is convergent and efficient with great stability. In addition, the running time of SRAF rises correspondingly as filter length is increasing. However, it has proved that filtering effect is improved with increasing filter length. Therefore, the trade-off between sidelobe level and time cost is very important.

Figure 8 shows the curves of PSLR, ISLR, and running time as the functions of filter length when SNR are infinite and $20 \mathrm{~dB}$. Figure 8A, B corresponds to correlation output of noise SAR and matched filtering output of general SAR, respectively. The left vertical axis denotes PSLR (ISLR) levels, and the right one represents time cost. In Figure 8A, as filter length varies from 300 to 3000 , the PSLR (ISLR) of output decreases from about $-30 \mathrm{~dB}(-18 \mathrm{~dB})$ to $-45 \mathrm{~dB}(-32 \mathrm{~dB})$. In Figure $8 \mathrm{~B}$, when filter length changes from 100 to 1200 , corresponding PSLR (ISLR) decreases from about $-30 \mathrm{~dB}(-21 \mathrm{~dB})$ to $-60 \mathrm{~dB}(-52 \mathrm{~dB})$. With increasing of filter length, the PSLR and ISLR curves are descending, but running time is ascending. Therefore, greater reduction of sidelobes is at the cost of rising running time. In these two applications, the best neutral filter lengths are 1500 to correlation output and 500 to matched filtering output.

\section{Conclusion}

The range resolution of SAR system is generally degraded due to high sidelobe level. Amplitude weighting is usually used to suppress sidelobes at the expense of mainlobe broadening and not applicable to noise SAR system. As a novel technique of sidelobe suppression, apodization filtering was originally proposed to control sidelobes for range response of noise radar. To improve the stability of this technique, a modified apodization filtering algorithm is suggested in this article. It can be applied to the range responses both of noise SAR and general SAR systems. The proposed method is SRAF with noticeable advantage of adequate stability for getting appropriate filtering vector. 

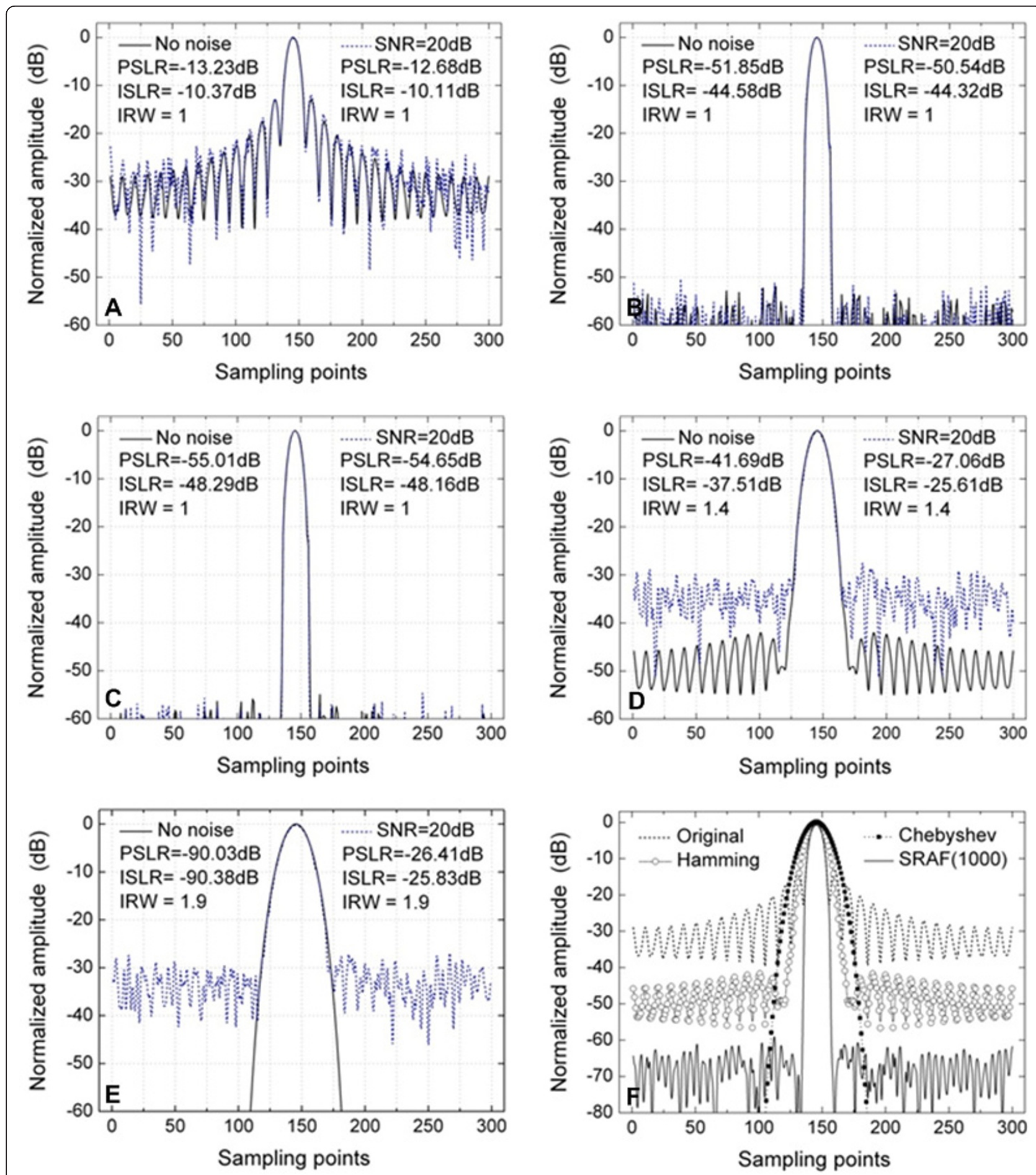

Figure 6 Matched filtering outputs before and after sidelobe suppression. The outputs relating to SNR $=\infty$ are denoted by black solid line; and the outputs relating to $S N R=20 \mathrm{~dB}$ are denoted by color dash line. (A) Original outputs without sidelobe suppression. (B) Outputs filtered by SRAF with filter length 500. (C) Outputs filtered by SRAF with filter length 800. (D) Outputs weighted by Hamming window. (E). Outputs weighted by Chebyshev window. (F) Performance comparison of algorithms $(\mathrm{SNR}=\infty)$.

There are three key steps of SRAF: getting coefficient matrix and desired response vector; performing ill-posed analysis; and solving equation for filtering vector.
Convolution kernel method is adopted to construct coefficient matrix; it makes the product between matrix $K$ and vector $\boldsymbol{F}$ be equivalent to the convolution of 


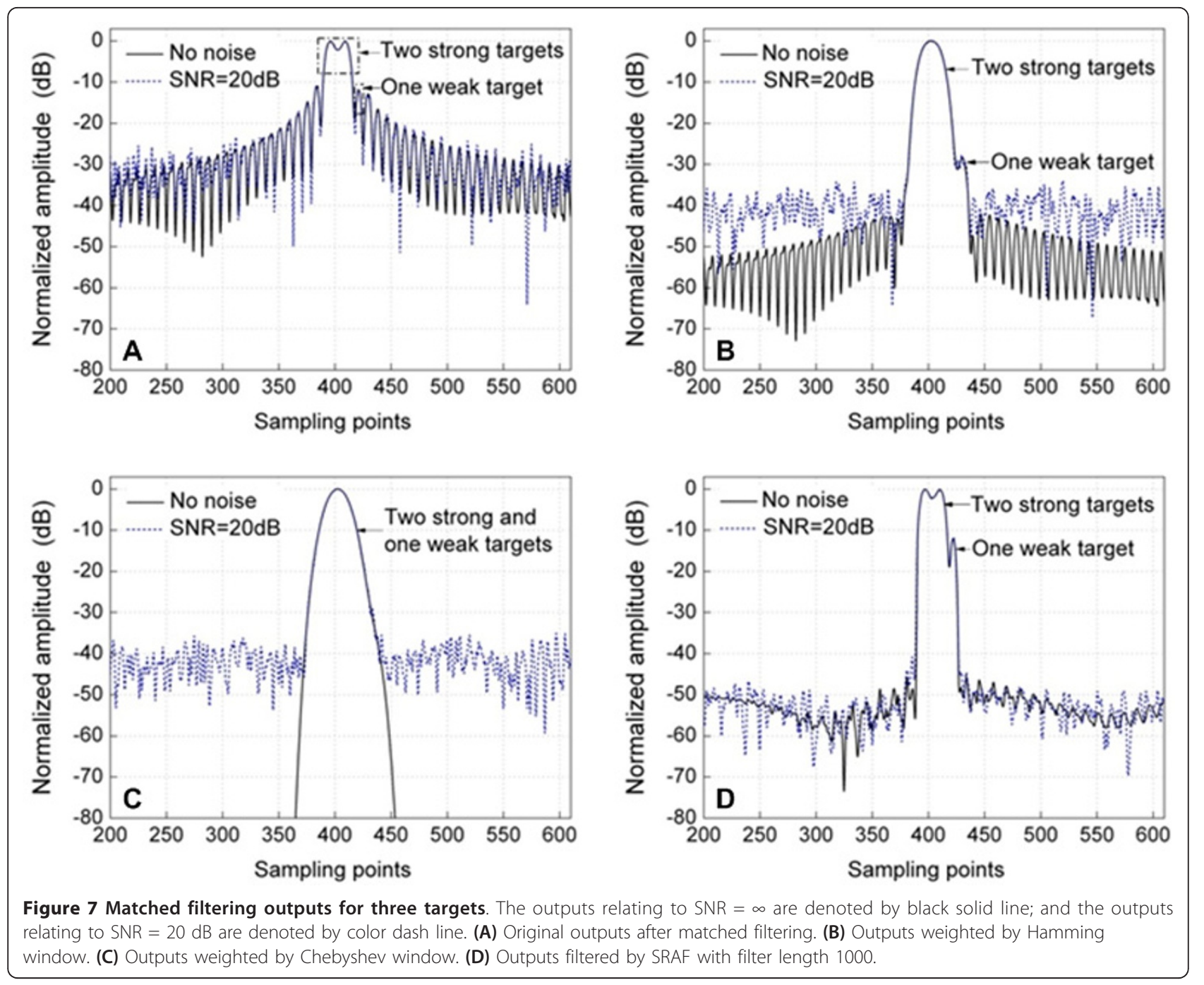

Equation (1). Spectral condition is introduced to estimate whether Equation (2) represents an ill-posed system; its value is large means that the equation is ill posed. TV method is implemented to solve Equation (2) for correct filtering vector because the least square method is not feasible to resolve ill-posed equation. With no restriction of solution to be smooth, TV ensures that solving equation process is stable enough to obtain appropriate filtering vector.

SRAF is executed to suppress range sidelobes of correlation output and matched filtering output for noise SAR and general SAR, respectively. Indeed, correct filtering vector can be obtained within several iterative steps. Sidelobe reductions of $12 \mathrm{~dB}$ and greater can be

Table 2 Singular values and spectrum conditions of $K$

\begin{tabular}{|c|c|c|c|c|c|c|c|c|c|c|c|}
\hline Noise SAR & Filter length & 300 & 600 & 900 & 1200 & 1500 & 1800 & 2100 & 2400 & 2700 & 3000 \\
\hline & $\sigma_{\max }$ & 14.02 & 17.46 & 18.19 & 20.03 & 19.58 & 20.26 & 20.21 & 20.77 & 20.35 & 20.86 \\
\hline & $\sigma_{\min }\left(10^{-10}\right)$ & 3.78 & 1.73 & 1.55 & 0.92 & 0.69 & 0.65 & 0.48 & 0.43 & 0.37 & 0.36 \\
\hline & $\kappa(\boldsymbol{K})\left(10^{10}\right)$ & 3.71 & 10.09 & 11.73 & 21.77 & 28.38 & 31.17 & 42.10 & 48.30 & 54.86 & 57.93 \\
\hline \multirow[t]{4}{*}{ General SAR } & Filter length & 300 & 400 & 500 & 600 & 700 & 800 & 900 & 1000 & 1100 & 1200 \\
\hline & $\sigma_{\max }$ & 22.57 & 23.19 & 23.55 & 23.76 & 23.91 & 23.99 & 24.07 & 24.12 & 24.16 & 24.19 \\
\hline & $\sigma_{\min }\left(10^{-10}\right)$ & 1.87 & 1.73 & 1.65 & 1.54 & 1.38 & 1.31 & 1.26 & 1.20 & 1.11 & 1.07 \\
\hline & $\kappa(\boldsymbol{K})\left(10^{10}\right)$ & 12.07 & 13.40 & 14.27 & 15.42 & 17.33 & 18.31 & 19.10 & 20.10 & 21.76 & 22.61 \\
\hline
\end{tabular}


Table 3 Iterative steps and running time of SRAF

\begin{tabular}{llllllllllll}
\hline Noise SAR & Filter length & $\mathbf{3 0 0}$ & $\mathbf{6 0 0}$ & $\mathbf{9 0 0}$ & $\mathbf{1 2 0 0}$ & $\mathbf{1 5 0 0}$ & $\mathbf{1 8 0 0}$ & $\mathbf{2 1 0 0}$ & $\mathbf{2 4 0 0}$ & $\mathbf{2 7 0 0}$ & $\mathbf{3 0 0 0}$ \\
\hline \multirow{6}{*}{ General SAR } & Iterative steps & 2 & 3 & 3 & 3 & 3 & 3 & 3 & 4 & 3 & 3 \\
& Running time (s) & 0.16 & 1.58 & 4.31 & 12.83 & 23.22 & 43.95 & 74.93 & 146.79 & 177.05 & 208.06 \\
& Filter length & 300 & 400 & 500 & 600 & 700 & 800 & 900 & 1000 & 1100 & 1200 \\
& Iterative steps & 5 & 4 & 5 & 8 & 8 & 5 & 10 & 8 & 7 & 7 \\
& Running time (s) & 2.35 & 5.01 & 14.73 & 46.57 & 74.51 & 70.29 & 200.73 & 216.53 & 245.39 & 324.28 \\
\hline
\end{tabular}
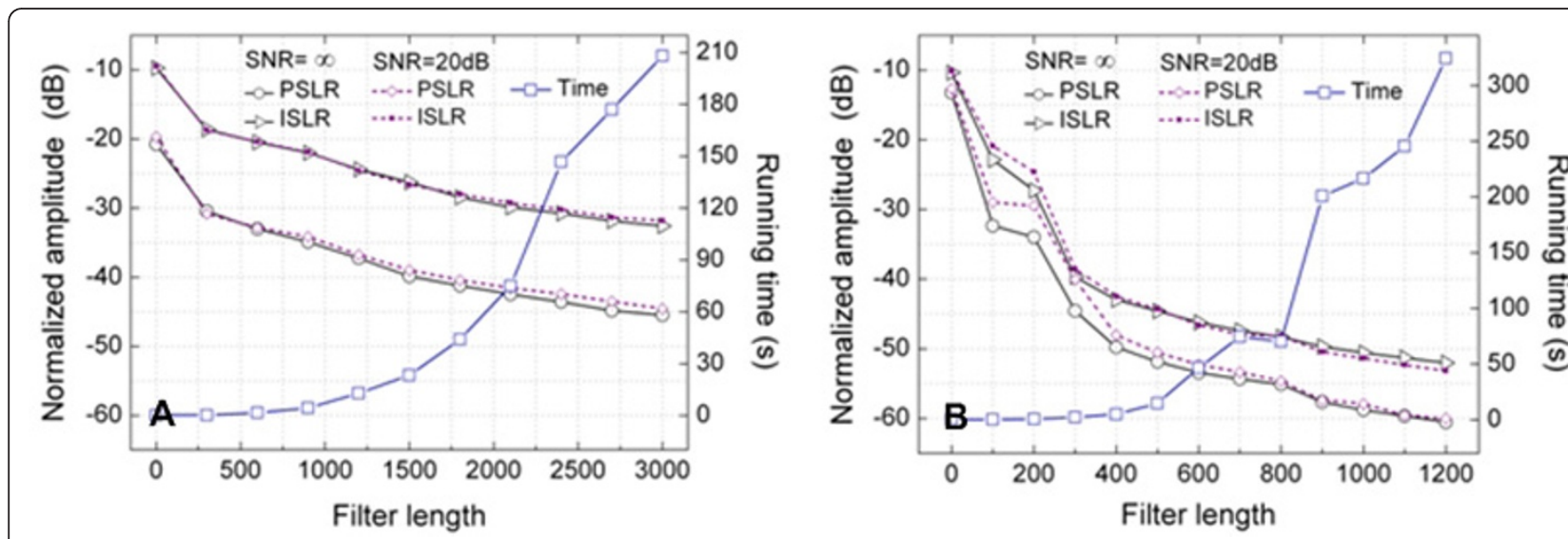

Figure 8 PSLR, ISLR, and running time curves depending upon filter length. The curves relating to SNR $=\infty$ are described by black solid line; and the curves relating to SNR $=20 \mathrm{~dB}$ are described by color short dash line. The curves of PSLR are denoted by symbol of hollow circle; the curves of ISRL are denoted by symbol of hollow triangle; and the curves of running time are denoted by symbol of hollow square. (A) Curves of correlation outputs. (B) Curves of matched filtering outputs.

achieved by this method; moreover, mainlobe energy is preserved well, which is contrast to obvious mainlobe broadening after amplitude weighting. With increasing filter length, the performance of sidelobe suppression is improved at the cost of increasing running time, so that it is important to select a balanced filter length. Noise disturbance is also considered in simulations. As SNR degenerates from infinite to $20 \mathrm{~dB}$, the performance of filtered outputs have no obvious degradation. In the cases of SNR $=-0.5$ and $-10 \mathrm{~dB}$, SRAF is still able to depress sidelobes effectively.

\section{Abbreviations}

ISLR: integrated sidelobe level ratio; LSS: least square solution; ORAF: original realization of apodization filtering; PSLR: peak sidelobe level ratio; SAR: synthetic aperture radar; SRAF: stable realization of apodization filtering; SVA: spatially variant apodization; SVD: singular value decomposition; TV: total variation.

\section{Acknowledgements}

This study was supported by the Department of Airborne Microwave Remote Sensing System of Institute of Electronics. We appreciate the assistance of Research Fellow GaoXin who provided many helpful comments.

\section{Author details}

'Institute of Electronics, Chinese Academy of Sciences, Beijing 100190, China ${ }^{2}$ Graduate University of Chinese Academy of Sciences, Beijing 100039, China

\section{Competing interests}

The authors declare that they have no competing interests.

Received: 23 November 2011 Accepted: 19 May 2012

Published: 19 May 2012

\section{References}

1. GW Davidson, IG Cumming, MR Ito, A chirp scaling approach for processing squint mode SAR data. IEEE Trans Aerosp Electron Syst. 32(1), 121-133 (1996)

2. IG Cumming, FH Wong, Digital Processing of Synthetic Aperture Radar Data: Algorithms and Implementation. Artech House, Norwood 36-168 (2005)

3. GS Liu, H Gu, WM Su, Development of random signal radars. IEEE Trans Aerosp Electron Syst. 35(3), 770-777 (1999). doi:10.1109/7.784050

4. RM Narayanan, XJ Xu, Principles and applications of coherent random noise radar technology. in Paper presented at the Proc. SPIE Conference on Noise in Devices and Circuits, Santa Fe, NM (12 May 2003)

5. SRJ Axelsson, Noise radar using random phase and frequency modulation. IEEE Trans Geosci Remote Sens. 42(11), 2370-2384 (2004)

6. RM Narayanan, Y Xu, PD Homffmeyer, JO Curtis, Design, performance, and applications of a coherent ultra-wide and random noise radar. Opt Eng. 37(6), 1855-1869 (1998). doi:10.1117/1.601699

7. IP Theron, EK Walton, S Gunawan, L Cai, Ultrawide-Band noise radar in the VHF/UHF band. IEEE Trans Antennas Propagat. 47(6), 1080-1084 (1999). doi:10.1109/8.777135

8. D Garmatyuk, RM Narayanan, Ultrawideband noise synthetic aperture radar: theory and experiment. in Paper presented at the IEEE Antennas Propagat Soc Int Symp 1999, Orlando, FL (11-16 July 1999)

9. D Tarchi, K Lukin, J Fortuny-Guasch, SAR imaging with noise radar. IEEE Trans Aerosp Electron Syst. 46(3), 1214-1225 (2010)

10. DC Bell, RM Narayanan, ISAR imaging using a coherent ultrawideband random noise radar system. Opt Eng. 40(11), 2612-2620 (2001). doi:10.1117/ 1.1413212 
11. XXu, RM Narayanan, FOPEN SAR imaging using UWB step-frequency and random noise waveforms. IEEE Trans Aerosp Electron Syst. 37(4), 1287-1300 (2001). doi:10.1109/7.976965

12. RM Narayanan, $X X \mathbf{X}, J A$ Henning, Radar penetration imaging using ultrawideband (UWB) random noise waveforms. IEE Proc Radar Sonar Navigat. 151(3), 143-148 (2004). doi:10.1049/ip-rsn:20040418

13. RM Narayanan, M Dawood, Doppler estimation using a coherent ultrawideband random noise radar. IEEE Trans Antennas Propagat. 48(6), 868-878 (2000). doi:10.1109/8.865218

14. H Wang, RM Narayanan, ZO Zhou, Through-Wall imaging of moving targets using UWB random noise radar. IEEE Antennas Wirel Propagat Lett. 8, 802-805 (2009)

15. CP Lai, RM Narayanan, Ultrawideband random noise radar design for throughwall surveillance. IEEE Trans Aerosp Electron Syst. 46(4), 1716-1730 (2010)

16. DS Garmatyuk, RM Narayanan, ECCM capabilities of an ultrawideband bandlimited random noise imaging radar. IEEE Trans Aerosp Electron Syst. 38(4), 1243-1255 (2002). doi:10.1109/TAES.2002.1145747

17. H Jiang, BC Zhang, YG Lin, W Hong, YR Wu, Random noise SAR based on compressed sensing. in Paper presented at the IEEE Geosic Remote Sensing Int Symp, Honolulu, HI (25-30 July 2010)

18. AH Nuttall, Some windows with very good sidelobe behavior. IEEE Trans Acoust Speech Signal Process. 29(1), 84-91 (1981). doi:10.1109/ TASSP.1981.1163506

19. RD Fry, DA Gray, CLEAN deconvolution for sidelobe suppression in random noise radar. in Paper presented at the Proc IEEE 2008 Radar Conf, Adelaide, SA-5 (2-5 September 2008)

20. HC Stankwitz, RJ Dallaire, JR Fienup, Nonlinear apodization for sidelobe control in SAR imagery. IEEE Trans Aerosp Electron Syst. 31(1), 267-279 (1995)

21. C Castillo-Rubio, S Llorente-Romano, M Burgos-Garcia, Robust SVA method for every sampling rate condition. IEEE Trans Aerosp Electron Syst. 43(2), 571-580 (2007)

22. $\mathrm{XXu}, \mathrm{RM}$ Narayanan, Range sidelobe suppression technique for coherent ultra wide-band random noise radar imaging. IEEE Trans Antennas Propagat. 49(12), 1836-1842 (2001). doi:10.1109/8.982467

23. M Hanke, PC Hansen, Regularization methods for large-scale problems. Surv Math Ind. 3, 253-315 (1993)

24. VA Morozov, On regularization of ill-posed problems and selection of regularization parameter. Comput Math Phys. 6(1), 170-175 (1996)

25. P Blomgren, TF Chan, P Mulet, CK Wong, Total variation image restoration: numerical methods and extension. in Paper presented at the IEEE Int Cof on Image Processing, Washington, DC (26-29 October 1997)

26. MY Zou, Deconvolution and Signal Recovery, (National Defence Industry Press, Beijing, 2001), pp. 242-251

27. MY Zou, X Zou, Global optimization: an auxiliary cost function approach IEEE Trans Syst Man Cybern A: Syst Humans. 30(3), 347-354 (2000). doi:10.1109/3468.844358

28. DM Strong, TF Chan, Relation of regularization parameter and scale in total variation based image denoising. http://www.math.ucla.edu/ imagers/ htmls/reports.html (1996). Accessed 6 Feb 2012

doi:10.1186/1687-6180-2012-112

Cite this article as: Wu et al.: A stable realization of apodization filtering applied to noise SAR and SAR range sidelobe suppression. EURASIP Journal on Advances in Signal Processing 2012 2012:112.

\section{Submit your manuscript to a SpringerOpen ${ }^{\mathcal{O}}$ journal and benefit from:}

- Convenient online submission

- Rigorous peer review

- Immediate publication on acceptance

- Open access: articles freely available online

- High visibility within the field

- Retaining the copyright to your article

Submit your next manuscript at $\gg$ springeropen.com 